\title{
Introduction: Vascular surgery comes of age
}

\author{
H. H. G. EASTCOTT \\ M.S., F.R.C.S. \\ St Mary's Hospital, London, W.2
}

TWENTY-ONE years ago the prophecies of Alexis Carrel began to come true. Based upon his work in the experimental animal laboratory 50 years before, they showed the great and wide-ranging possibilities of vascular reconstruction. But it was not until 1949 that these discoveries were applied to patients, when Robert Gross and his team in Boston successfully inserted homografts into the aorta of several young people with coarctation. Then followed a great rush of clinical work further confirming the safety and effectiveness of the arterial homograft in many differing clinical situations. A new dimension in surgical technique had been opened up and researchers were quick to adapt it to that other great vision of Carrel's, the transplantation of living organs. Indeed as early as 1954 there was clear distinction between static and vital grafts, and not long after this, the remarkably easy adaptation of plastic textiles to the functions of the dead, static homograft soon led to the adoption of the cloth tube graft for many new and ingenious projects in virtually any part of the trunk and limb roots that might be affected by arterial disease. Foremost in this new field was Michael DeBakey whose experience and expertise soon became legendary, and the pattern for established good practice all over the world.

Yet perhaps the most important outcome of all this surgical handicraft and industry was an unexpected one. With the incalculable help of safe, clear arteriography in routine everyday use, surgeons began to look beyond the effects of degenerative disease and other kinds of damage to the large arteries that they had been repairing, and to consider the mechanism of the production and development of these lesions and, since the pathologists had mostly kept away from the topic, even to ponder upon the causes. It now seems to be emerging that occlusive vascular disease on both the arterial and the venous sides of the circulation is a thromboembolic process, with rising resistance not only from narrowing of main channels, but also in the microcirculation as a result of repeated minor embolic detachments that can be readily observed in the small vessels of the retina, the tips of the digits, as well as from changes in the urine that suggested that similar small infarcts might also be occurring in the kidney. Amputated limbs showed lesions throughout the muscle layers, with the same pattern of cholesterol clefts within the lumen of arteries much smaller tham those affected by the parent atheromatous layering whose occasional eruption had discharged ths material into the periphery.

Adjustments in the local circulation at the sit of these blockages depends upon a natural capacit to form collateral pathways, a factor which varie with age, extent and duration of the disease as we as the condition of the blood and the heart itself Whether or not an occlusion can be overcome by the returning tide of recovering flow before gangrenif develops at the periphery, may therefore depen more upon the haemodynamic effects of these variables than the anatomy of the obstruction itself: Through the several articles that appear in this issu of the Journal runs this trend of thought, and the judgments of diagnosis and case management wilb be seen to be profoundly affected by it. Measurenent of flow by electromagnetic meters at operation, cinfirming the more qualitative findings of the Dop per ultrasonic probe for transcutaneous examination of both arteries and veins is yielding at last the scientifi. answers to questions that vascular surgeons havo long been asking. The 'steal' phenomenon, by which the arterial supply to a part of the body can be t $\overrightarrow{\mathrm{Q}}$ the detriment of neighbouring regions, first recog: nized in the subclavian as a cause of intermitten cerebral ischaemia, is now believed to apply to many if not most disorders of arterial occlusion in the heart, the brain itself. It has long been known that muscle hyperaemia after exercise in claudicators wit $\underline{\underline{B}}$ an obstructed main artery can lead to the disappeai ance of ankle pulses that were easily palpable at rest while the foot becomes pale at the same time. Yof vasodilators with systemic action are still prescribe for patients in whom one affected limb has a higheg resistance to flow than the other three in which the drug will almost certainly steal blood from the obstructed limb.

We can now see that the rational treatment of vascular disease stands more securely than evef before, thanks largely to the contributions of surgeons such as these who write in this issue, anf who have shown and proved the importance not onles of overcoming the local lesion by their technicat expertise, but also by a due regard for the primo importance of the pump and the fluid with whic their pipes are intimately concerned. 